\title{
Isotropic Elastoplasticity Fully Coupled with Non-Local Damage
}

\author{
Madjid Almansbaa ${ }^{1,2}$, Khémais Saanouni ${ }^{1}$, Nacer Eddine Hannachi ${ }^{2}$ \\ ${ }^{1}$ I. C. D. LASMIS, Université de Technologie de Troyes 12, Rue Marie Curie, Troyes Cedex, France \\ ${ }^{2}$ LAMOMS, Université Mouloud Mammeri Tizi Ouzou, BP 17 Route de Hasnaoua Tizi Ouzou, \\ Tizi Ouzou, Algérie \\ E-mail:almansbm@mail.ummto.dz,khemais.saanouni@utt.fr,hannachina@yahoo.fr \\ Received December 28, 2009; revised February 23, 2010; accepted February 26, 2010
}

\begin{abstract}
This paper presents a simple damage-gradient based elastoplastic model with non linear isotropic hardening in order to regularize the associated initial and boundary value problem (IBVP). Using the total energy equivalence hypothesis, fully coupled constitutive equations are used to describe the non local damage induced softening leading to a mesh independent solution. An additional partial differential equation governing the evolution of the non local isotropic damage is added to the classical equilibrium equations and associated weak forms derived. This leads to discretized IBVP governed by two algebric systems. The first one, associated with equilibrium equations, is highly non linear and can be solved by an iterative Newton Raphson method. The second one, related to the non local damage, is a linear algebric system and can be solved directly to compute the non local damage variable at each load increment. Two fields, linear interpolation triangular element with additional degree of freedom is terms of the non local damage variable $\bar{D}$ is constructed. The non local damage variable $\bar{D}$ is then transferred from mesh nodes to the quadrature (or Gauss) points to affect strongly the elastoplastic behavior. Two simple 2D examples are worked out in order to investigate the ability of proposed approach to deliver a mesh independent solution in the softening stage.
\end{abstract}

Keywords: Elastoplastic, Damage Behaviour Coupling, Isotropic Hardening, Damage Gradient, Finis Elements

\section{Introduction}

It is well known that the local constitutive equation exhibiting an induced strain softening which succeeds to the positive strain hardening, leads inevitably to more or less strain localization. Strain localization refers to the emergence of finite narrow bands inside which the plastic flow localizes while the remaining part of the deforming body is elastically unloaded. However, it has been shown in several published works, that the numerical solution (using FEM) of this class of dissipation problems exhibits a high sensitivity to the space and time discretization [1-7]. Among these problems, the fully coupled constitutive equations accounting for positive hardening and damage induced softening (or negative hardening) are extensively studied during the last decade [8].

To regularize the solution of these problems, leads to incorporate some effects of characteristic lengths of the materials microstructure into constitutive models via the mechanics of generalized continue as the higher grade continues [9-12] or higher order continua [13-15]. In these approaches, the stress at a given material point depends on additional degrees of freedom as well their higher order spatial derivatives or gradients. The must recent and comprehensive presentation of the mechanics of generalized continua can be found in [13-15]. In this work a simple damage-gradient based elastoplastic constitutive equations accounting for the non linear isotropic hardening fully coupled with non local ductile damage variable is presented. Inspired by the works of $[6,16]$ an implicit damage non locality equation is added to the classical equilibrium equations in order to derive a two functional variational formulation with additional degree of freedom which is the non local damage variable. The non local damage variable is introduced on the state and dissipation potentials thanks to the effective state variables based on the assumption of total energy equivalence [17]. A new finite element is then formulated with the non local damage as an additional degree of freedom. 
This leads to two algebric systems, one highly non linear and the other is quite linear giving a non local damage at each load increment. Some simple examples are performed in order to show the ability of the proposed approach to avoid the mesh dependency of the solution of the IBVP.

\section{On Isotropic Elasto-Plasticity with Damage-Gradient}

In previous works $[8,18]$ the authors have proposed advanced constitutive equations for sheet or balk metal forming accounting for thermal effect, elasto (visco) plasticity, mixed non linear hardening, isotropic ductile damage and contact with friction. These fully coupled multiphysics models has been formulating, in the framework of the thermodynamics of irreversible processes assuming a fully local theory in which only the first gradient of the displacement is required. In this work, a non local damage variable $(\bar{D})$ is introduced in these constitutive equations replacing the classical local damage variable $(D)$ by the non local damage variable $(\bar{D})$ solution of the following partial differential equation [6]:

$$
\left\{\begin{array}{lll}
\bar{D}-\omega \operatorname{div}(\vec{\nabla} \bar{D})=0 & \text { in } \Omega_{D} \quad \text { (a) } \\
(\overline{\operatorname{grad}} \bar{D}) \cdot \vec{n}=0 & \text { in } \Gamma_{\bar{D}} \quad \text { (b) }
\end{array}\right.
$$

The Equation (1(b)) represents the Neumann type boundary condition prescribed on the boundary $\Gamma_{\bar{D}}$ of the damaged volume $\Omega_{D}$ and $\omega$ is homogeneous to a length squared and plays the role of an internal characteristic length of material, governing the non-locality of the damage field. This leads to introduce the damage gradient in the classical local constitutive equations in order to regularize the initial boundary value problem (IBVP) with respect to the space and time discretization. For the sake of simplicity we limit resolves to the fully isotropic and isothermal elastoplasticity assuming the von Mises yield function and non linear isotropic hardening assuming the small strain hypothesis.

\subsection{Constitutive Model for Plasticity with Isotropic Hardening and Non Local Damage}

Following the idea by [19], the non local damage variable is computed from Equation (1) and introduced into the local constitutive equations simply by replacing the local damage variable $(D)$ by $(\bar{D})$. The complete set of these constitutive equations fully coupled with non local damage variable is then given by:

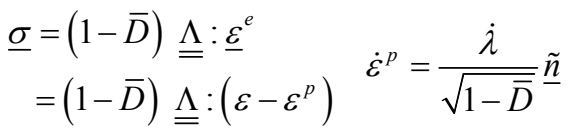

$$
\begin{aligned}
& R=(1-\bar{D}) Q r \quad \dot{r}=\frac{\dot{\lambda}}{\sqrt{1-\bar{D}}}(1-b \mathrm{r} \sqrt{1-\bar{D}}) \\
& Y=\frac{1}{2} \varepsilon^{e}: \Lambda: \varepsilon^{e}+\frac{1}{2} Q r^{2} \quad \dot{D}=\frac{\dot{\lambda}}{(1-\bar{D})^{\beta}}\left\langle\frac{Y-Y_{0}}{S}\right\rangle^{s}
\end{aligned}
$$

where " $\underline{\sigma}$ " is the Cauchy's stress tensor, $\underline{\underline{\Lambda}}$ is the stiffness tensor, " $\varepsilon^{e}$ " the elastic strain tensor, " $\varepsilon^{p}$ " is the plastic strain, $R$ and $r$ are respectively the isotropic stress and its isotropic strain, " $Q$ " is the isotropic hardening modulus and " $b$ " is non-linearity parameter of the isotropic hardening. " $Y$ " the thermodynamic force associated to the local damage " $D$ " which resolves according to Equation (4(b)), in which $S, s, \beta$ and $Y_{0}$ are the ductile damage parameters. The deviatoric tensors $\underline{n}$ and $\underline{\tilde{n}}$ define the outward unit normal to the yield function $f=0$ in the stress and effective stress spaces respectively. They are defined by:

$$
\underline{n}=\frac{\partial f}{\partial \sigma}=\frac{3}{2} \frac{1}{\sqrt{1-\bar{D}}} \frac{\underline{S}}{\|\underline{\sigma}\|}=\frac{1}{\sqrt{1-\bar{D}}} \frac{\partial f}{\partial \underline{\tilde{\sigma}}}=\frac{\underline{\tilde{n}}}{\sqrt{1-\bar{D}}}
$$

In these equations " $\dot{\lambda}$ " is the classical plastic multiplier derived from the consistency condition applied to the yield function:

$$
f=\frac{\|\underline{\sigma}\|-R}{\sqrt{1-\bar{D}}}-\sigma_{y}
$$

in which " $\sigma_{y}$ " is the flow stress in simple tension and $\|\underline{\sigma}\|=\sqrt{\frac{3}{2} \underline{S}: \underline{S}}$ is the von Mises equivalent stress: $\left(\underline{S}=\underline{\sigma}-\frac{1}{3} \operatorname{tr}(\underline{\sigma}) \cdot \underline{1}\right)$. If the analytic form of $\dot{\lambda}$ is deduced from the consistency condition $(\dot{f}=0$ if $f=0)$, one can obtain $(\dot{\bar{D}}=\dot{D})$ :

$$
\dot{\lambda}= \begin{cases}\frac{1}{H_{p D}}\left\langle\frac{3 \mu_{e} \sqrt{1-\bar{D}} \underline{S}: \underline{\dot{\varepsilon}}}{\|\underline{S}\|}\right\rangle & \text { if } \dot{f}=0 \text { and } f=0 \\ 0 & \text { else where }\end{cases}
$$

where $\quad H_{p D}=3 \mu_{e}+(1-\bar{D}) Q-b R \sqrt{1-\bar{D}}+\hat{Y}\left(\frac{R}{\sqrt{1-\bar{D}}}+\frac{\sigma_{y}}{2}\right)$ is the elastoplastic hardening modulus. With $\hat{Y}=$ $\frac{1}{(1-\bar{D})^{\beta}}\left\langle\frac{Y-Y_{0}}{S}\right\rangle^{s}$. From numerical point of view, Equation (7) is not needed since $\Delta \lambda=\dot{\lambda} \Delta t$ is taken as the 
principal unknown to be computed from $f_{t+\Delta t}=0$ at the end of each time increment $\Delta t=t_{n+1}-t_{n}$.

\section{Numerical Aspects}

As discussed above the initial and boundary value problem is driven by the partial differential equations (PDEs) describing both the equilibrium problem (the inertia being neglected) and the damage non locality equation (Equation (1)):

$$
\left\{\begin{array}{llll}
\overrightarrow{\operatorname{div}}(\underline{\sigma})+\vec{f}_{v}=\overrightarrow{0} & \text { in } \Omega & \text { (a) } \\
\underline{\sigma} \cdot \vec{n}=\vec{F} & \text { in } & \Gamma_{\mathrm{F}} & \text { (b) } \\
\vec{U}=\overrightarrow{\bar{u}} & \text { in } & \Gamma_{\mathrm{u}} & \text { (c) }
\end{array}\right.
$$

where $\vec{f}_{v}$ is the body forces vector. $\vec{n}$ is the outward normal to the boundary $\Gamma_{F}$ where the forces vectors $\vec{F}$ is prescribed; while $\Gamma_{u}$ is the boundary of the $\Omega$ where the displacement $\overrightarrow{\vec{u}}$ vector is prescribed $\left(\Gamma_{u} \cup \Gamma_{F}=\Gamma\right.$ "the boundary of $\Omega$ and $\left.\Gamma_{u} \cap \Gamma_{F}=\varnothing\right)$.

Both Equation (1) and Equation (8) will be solved using the "displacement" based Galerkin finite element method is discussed here after.

\subsection{Variation Formulation and Global Resolution Scheme}

Let $\delta \vec{u}$ the virtual displacement vector and $\delta \bar{D}$ the virtual non local damage both compatible with the boundary conditions (i.e. kinematically admissible (K.A)). The weak forms associated with Equation (1) and Equation (8) are easily deduced:

$$
\left\{\begin{array}{cc}
I_{u}(\vec{u}, \delta \overrightarrow{\mathrm{u}})=-\int_{\Omega} \sigma: \vec{\nabla}(\overrightarrow{\delta u}) d \Omega+ & \\
\int_{\Omega} \overrightarrow{\delta u} f_{v} d \Omega+\int_{\Gamma_{F}} \overrightarrow{\delta u} \cdot \vec{F} \cdot d s=0 & \forall \delta \overrightarrow{\mathrm{u}} \mathrm{K} . \mathrm{A} \\
I_{\bar{D}}(\bar{D}, \delta \overline{\mathrm{D}})=-\int_{\Omega_{D}}(\omega \overrightarrow{\nabla \vec{D}} \cdot \overrightarrow{\nabla \delta \vec{D}}+\bar{D} \delta \bar{D}) d \Omega+ \\
\int_{\Omega_{D}} D \delta \bar{D} d \Omega=0 & \forall \delta \overline{\mathrm{D}} \mathrm{K} . \mathrm{A}
\end{array}\right.
$$

Since the domain $\Omega$ is discretized into many sub-domains (or finites elements) $\Omega^{e}$, then the elementary weak forms written on each element $(e)$ lead to:

$$
\left\{\begin{aligned}
I_{u}^{e}\left(\vec{u}^{e}, \delta \overrightarrow{\mathrm{u}}^{e}\right)= & -\int_{\Omega_{e}} \underline{\sigma}: \vec{\nabla}\left(\overrightarrow{\delta u^{e}}\right) d \Omega_{e}+ \\
& \int_{\Gamma_{F}} \overrightarrow{\delta u^{e}} \vec{F} d s_{e}+\int_{\Omega_{e}} f_{v} \overrightarrow{\delta u^{e}} d \Omega_{e} \\
I_{\bar{D}}^{e}\left(\bar{D}^{e}, \delta \overline{\mathrm{D}}^{e}\right) & =-\int_{\Omega_{D}^{e}}\left(\omega \overrightarrow{\nabla \vec{D}} \overrightarrow{\nabla \delta \bar{D}^{e}}+\bar{D} \delta \bar{D}^{e}\right) d \Omega \\
& +\int_{\Omega_{D}^{e}} D \delta \bar{D}^{e} d \Omega_{e}
\end{aligned}\right.
$$

Consequently, the system (9) can be written under the following discretized when the do main $\Omega$ is discretized into "Nele" elements from:

$$
\left\{\begin{array}{l}
I_{u}=\sum_{e=1}^{\text {Nele }} I_{u}^{e} \quad \forall \delta \overrightarrow{\mathrm{u}} \quad \text { K.A. } \\
I_{\bar{D}}=\sum_{e=1}^{\text {Nele }} I_{\bar{D}}^{e} \quad \forall \delta \overline{\mathrm{D}} \quad \text { K.A. }
\end{array}\right.
$$

By using the Bubnov-Galerkin method, the real and virtual quantities are approximated over each finite element (e) according to (the matrix notations will he used):

$$
\left\{\begin{array}{cl}
\left\{u^{e}\right\}=\left[N_{u}^{e}\right]\left\{u_{n}^{e}\right\} & \left\{\delta u^{e}\right\}=\left[N_{u}^{e}\right]\left\{\delta u_{n}^{e}\right\} \\
\left\{\bar{D}^{e}\right\}=\left[N_{\bar{D}}^{e}\right]\left\{\bar{D}_{n}^{e}\right\} & \left\{\delta \bar{D}^{e}\right\}=\left[N_{\bar{D}}^{e}\right]\left\{\delta \bar{D}_{n}^{e}\right\}
\end{array}\right.
$$

where $\left[N_{u}^{e}\right]$ and $\left[N_{\bar{D}}^{e}\right]$ are the matrices of the shape functions relative to the nodal unknown $\left\{u^{e}\right\}$ and $\left\{\bar{D}^{e}\right\}$. Their first gradients are then deduced:

$$
\begin{aligned}
& \left\{\begin{array}{l}
\left\{\varepsilon^{e}\right\}=\left[\frac{\partial N_{u}^{e}}{\partial x_{j}}\right]\left\{u_{n}^{e}\right\}=\left[B_{u}^{e}\right]\left\{u_{n}^{e}\right\} \\
\left\{\frac{\partial \bar{D}^{e}}{\partial x}\right\}=\left[\frac{\partial N_{\bar{D}}^{e}}{\partial x_{j}}\right]\left\{\bar{D}_{n}^{e}\right\}=\left[B_{\bar{D}}^{e}\right]\left\{\bar{D}_{n}^{e}\right\}
\end{array}\right. \\
& \left\{\begin{array}{l}
\left\{\delta \varepsilon^{e}\right\}=\left[\frac{\partial N_{u}^{e}}{\partial x_{j}}\right]\left\{\delta u_{n}^{e}\right\}=\left[B_{u}^{e}\right]\left\{\delta u_{n}^{e}\right\} \\
\left\{\frac{\partial \delta \bar{D}^{e}}{\partial x}\right\}=\left[\frac{\partial N_{\bar{D}}^{e}}{\partial x_{j}}\right]\left\{\delta \bar{D}_{n}^{e}\right\}=\left[B_{\bar{D}}^{e}\right]\left\{\delta \bar{D}_{n}^{e}\right\}
\end{array}\right.
\end{aligned}
$$

Introducing Equation (12) and Equation (13) in Equation (10) leads to:

$$
\left\{\begin{array}{c}
I_{u}^{e}\left(u^{e}\right)=\left\langle\delta u_{n}^{e}\right\rangle\left[\begin{array}{l}
-\int_{\Omega_{e}}\left[B_{u}^{e}\right]\{\sigma\} \cdot d \Omega_{e}+ \\
\int_{\Gamma_{e}}\left[N_{u}^{e}\right]^{T} \cdot\{\vec{T}\} \cdot d s_{e}+\int_{\Omega_{e}}\left[N_{u}^{e}\right]^{T}\{f\} d \Omega_{e}
\end{array}\right] \\
I_{\bar{D}}^{e}\left(\bar{D}^{e}\right)=\left\langle\delta \bar{D}_{n}^{e}\right\rangle\left[\begin{array}{l}
-\int_{\Omega_{e}}\left(\left[B_{\bar{D}}^{e}\right]^{T}[\omega]\left[B_{\bar{D}}^{e}\right]+\left[N_{\bar{D}}^{e}\right]\right)\left\{\bar{D}_{k}\right\} d \Omega \\
+\int_{\Omega_{e}}\left[N_{\bar{D}}^{e}\right]^{T}\{D\} d \Omega_{e}
\end{array}\right]
\end{array}\right.
$$

In Equation (14(b)) $[\omega]$ is the diagonal matrix of internal lengths.

$$
[\omega]=\left[\begin{array}{cccc}
l^{2} & 0 & . . & 0 \\
: & l^{2} & . . & 0 \\
: & . . & : & : \\
0 & 0 & . . & l^{2}
\end{array}\right]
$$


After the standard assembly operation, the following algebric system written at $t_{n+1}$ is obtained:

$$
\left\{\begin{array}{l}
\{R\}_{n+1}=\left[\sum_{e}\left(\left\{F^{e}\right\}_{\mathrm{int}}-\left\{F^{e}\right\}_{\text {ext }}\right)\right]_{n+1}=0 \\
\{H\}_{n+1}=\left[\sum_{e}\left(\left[K_{\bar{D} \bar{D}}^{e}\right]\{\bar{D}\}-\left\{F_{D}^{e}\right\}\right)\right]_{n+1}=0
\end{array}\right.
$$

where the integrals entering the matrix $\left[K_{\bar{D} \bar{D}}^{e}\right]$ and the vectors $\left\{F^{e}\right\}_{\text {int }},\left\{F^{e}\right\}_{e x t}$ and $\left\{F_{\bar{D}}^{e}\right\}$ can be written with the help of Newton integration method, as follows:

$$
\begin{aligned}
& \left\{F^{e}\right\}_{\mathrm{int}}=\sum_{N p g} \pi_{j}\left[B_{u}^{e}\right]^{T}\{\underline{\sigma}\} J \\
& \left\{F^{e}\right\}_{\text {ext }}=\sum_{N p g} \pi_{j}\left[N_{u}^{e}\right]^{T}\{f\} J+\sum_{N p g} \pi_{j}\left[N_{u}^{e}\right]^{T}\{T\} J_{s}
\end{aligned}
$$

$$
\begin{aligned}
& {\left[K_{\bar{D} \bar{D}}^{e}\right]=\sum_{N p g} \pi_{j}\left(\left[B_{\bar{D}}^{e}\right]^{T}[\omega]\left[B_{\bar{D}}^{e}\right]+\left[N_{\bar{D}}^{e}\right]^{T}\left[N_{\bar{D}}^{e}\right]\right)} \\
& \left\{F_{\bar{D}}^{e}\right\}=\sum_{N p g} \pi_{j}\left[N_{\bar{D}}^{e}\right]^{T}\{D\} J
\end{aligned}
$$

From Equation (16) it is worth noting that if the first Equation (16(a)) is highly non linear and should be solved iteratively, the second one Equation (16(b)) is perfectly linear an can be solved directly without any iterative procedure [20]. For this end, a 2D plane strain simple linear (triangle) element is constructed with three degrees of freedom (dof) per node namely two displacement components $(\mathrm{u}, \mathrm{v})$ and the non local damage $(\bar{D})$. Since the dof's (u,v) are independent from dof $(\bar{D})$, similar linear approximation is assumed for both $(\mathrm{u}, \mathrm{v})$ and $(\bar{D})$. Accordingly a very classical triangular isoparametric element with a single integration point is used. The Figure 1 summarizes the resolution scheme used

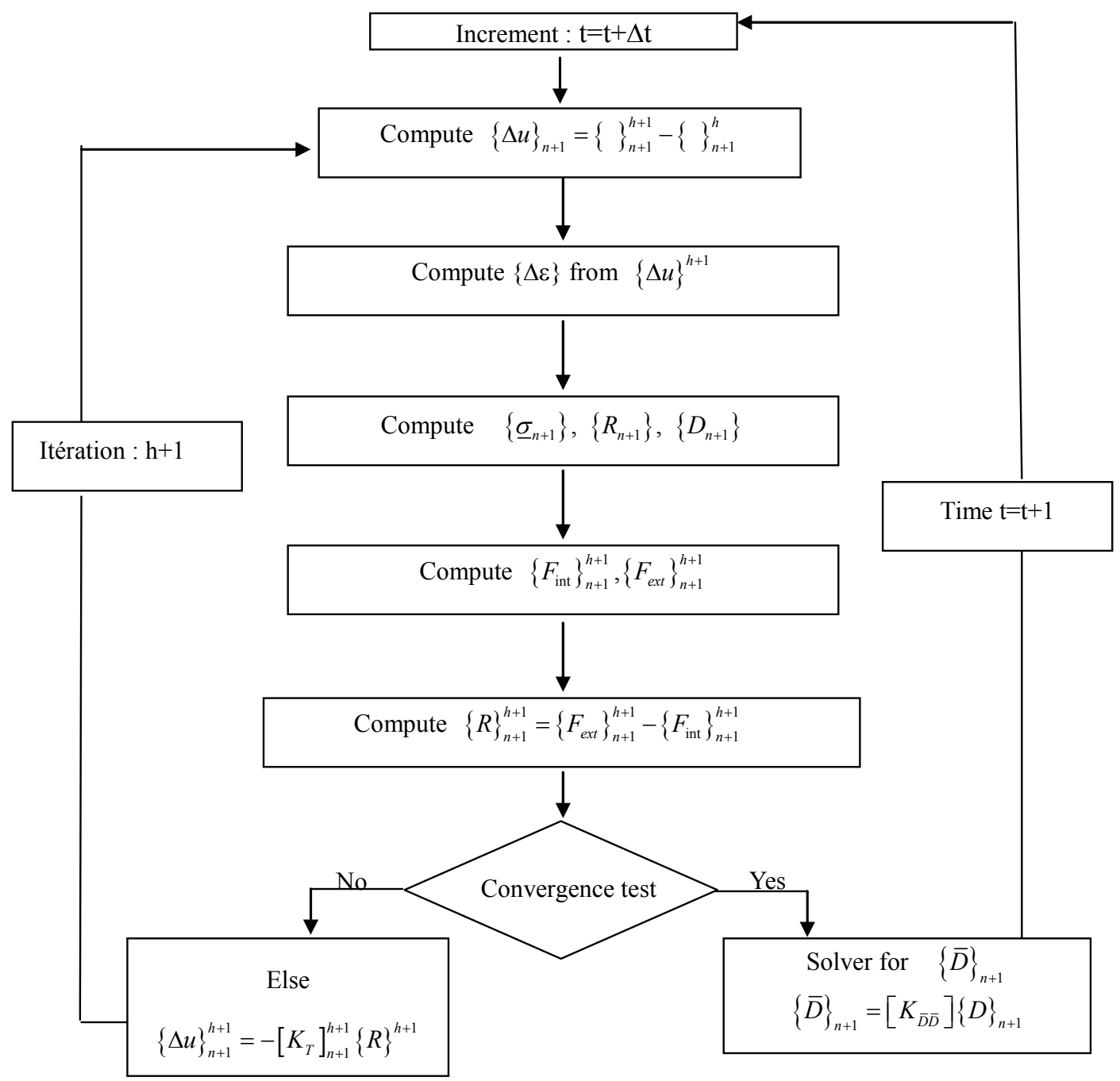

Figure 1. Flow chart of the global resolution scheme. 
in this work to solve Equation (16).

\subsection{Local Integration Scheme}

At each time $t_{n+1}$, the calculation of the internal forces $\left\{F^{e}\right\}_{\text {int }}$ in Equation (17(a)) and $\left\{F_{\bar{D}}^{e}\right\}$ in Equation (18(b)) needs the computation of the stress $\{\sigma\}_{n+1}$ and local damage $\{D\}_{n+1}$. This is done through the numerical integration of the fully coupled constitutive equations presented above Equation (2) to Equation (4). The standard elastic prediction and plastic correction algorithm $[8,21,22]$ is used for computation of the stress tensor together with the other state variables of the model.

\subsubsection{Elastic Prediction}

Elastic prediction stage consists to assume that the given total strain increment is elastic without any dissipation (i.e. $\Delta \lambda=0$.). This leads to define the "trial" stress as:

$$
\underline{\sigma}_{n+1}^{*}=\left(1-\bar{D}_{n}\right) \underline{\Lambda}: \underline{\varepsilon}_{n+1}^{*}
$$

where $\underline{\varepsilon}_{n+1}^{*}=\underline{\varepsilon}_{n+1}+\Delta \underline{\varepsilon}-\underline{\varepsilon}_{n}^{p}$ is the known trial strain supposed as purely elastic. The "trial" yield function is then defined by:

$$
f_{n+1}^{*}=\frac{\left\|\underline{\sigma}_{n+1}^{*}\right\|-R_{n}}{\sqrt{1-\bar{D}_{n}}}-\sigma_{y}
$$

The non local damage " $\bar{D}$ " at each integration point is obtained by linear interpolation from the nodes and is kept contort equal to " $\bar{D}_{n}$ " during the iterative resolution of the equilibrium equations:

If $f_{n+1}^{*}<0$ the solution is effectively elastic and the solution is:

$$
\underline{\sigma}_{n+1}=\underline{\sigma}_{n+1}^{*}, \quad R_{n+1}=R_{n}, \quad \underline{\varepsilon}_{n+1}^{p}=\underline{\varepsilon}_{n}^{p}, \quad D_{n+1}=D_{n}
$$

If $f_{n+1}^{*}>0$, the trial solution should corrected in order to fulfill the yield criterion.

\subsubsection{Plastic Correction}

For the model used here in the plastic flow is governed by the unique scalar equation $f_{n+1}\left(\Delta \lambda, \bar{D}_{n}\right)=0$ in which $\bar{D}_{n}$ is known at each integration point by interpolation of nodal values.

The problem is then to calculate $\underline{\sigma}_{n+1}, R_{n+1}, \varepsilon_{n+1}^{p}$, $D_{n+1}$ which are plastically admissible, i.e., verifying at $t_{n+1}$ :

$$
f_{n+1}=\frac{\left\|\sigma_{n+1}\right\|-R_{n+1}}{\sqrt{1-\bar{D}_{n}}}-\sigma_{y}=0
$$

By using the time discretisation scheme of the constitutive equations one can obtain:

$$
\begin{gathered}
\underline{\sigma}_{n+1}=\underline{\sigma}_{n+1}^{*}-2 \mu \sqrt{1-\bar{D}_{n}} \Delta \lambda \underline{\tilde{n}}_{n+1} \\
R_{n+1}=\frac{R_{n}+Q \sqrt{1-\bar{D}_{n}} \Delta \lambda}{1+b \Delta \lambda} \\
\text { with } \quad R_{n}=Q\left(1-\bar{D}_{n-1}\right) r_{n}
\end{gathered}
$$

By locking the deviatoric part of $\sigma_{n+1}$ from Equation (23) it comes:

$$
\underline{S}_{n+1}=\underline{S}_{n+1}^{*}-2 \mu \sqrt{1-\bar{D}_{n}} \Delta \lambda \underline{\tilde{n}}_{n+1}
$$

On the other the normal's to the yield surface $f_{n+1}$ and "trial" yield surface $f^{*}$ are given by:

$$
\underline{\tilde{n}}_{n+1}=\frac{3}{2} \frac{\underline{S}_{n+1}}{\left\|\underline{\sigma}_{n+1}\right\|} \text { and } \underline{\tilde{n}}_{n+1}^{*}=\frac{3}{2} \frac{\underline{S}_{n+1}^{*}}{\left\|\underline{\sigma}_{n+1}^{*}\right\|}
$$

Combining Equation (25) and Equation (26) leads to:

$$
\left\|\underline{\sigma}_{n+1}\right\| \underline{\tilde{n}}_{n+1}=\left\|\underline{\sigma}_{n+1}^{*}\right\| \underline{\tilde{n}}_{n+1}^{*}-3 \mu \sqrt{1-\bar{D}_{n}} \Delta \lambda \underline{\tilde{n}}_{n+1}
$$

This equation leads to:

$$
\underline{\tilde{n}}_{n+1}=\underline{\tilde{n}}_{n+1}^{*} \text { i.e. return mapping }
$$

And:

$$
\left\|\underline{\sigma}_{n+1}\right\|=\left\|\underline{\sigma}_{n+1}^{*}\right\|-3 \mu \sqrt{1-\bar{D}_{n}} \Delta \lambda
$$

Using Equation (29) together with Equation (22) leads to a single scalar equation with $\Delta \lambda$ as a single unknown:

$$
\begin{gathered}
\underline{\varepsilon}_{n+1}^{p}=\underline{\varepsilon}_{n}^{p}+\frac{\Delta \lambda \underline{\tilde{n}}_{n+1}}{\sqrt{1-\bar{D}_{n}}} \\
D_{n+1}=D_{n}+\frac{\Delta \lambda}{\left(1-\bar{D}_{n+1}\right)^{\beta}}\left\langle\frac{Y_{n+1}-Y_{0}}{S}\right\rangle^{s}
\end{gathered}
$$

where:

$$
\begin{aligned}
Y_{n+1}= & \frac{1}{2}\left(\varepsilon_{n+1}^{*}-\frac{\Delta \lambda}{\sqrt{1-\bar{D}_{n}}} \underline{n}\right): \underline{\Lambda}:\left(\varepsilon_{n+1}^{*}-\frac{\Delta \lambda}{\sqrt{1-\bar{D}_{n}}} \underline{n}\right)+ \\
& \frac{1}{2} \frac{Q}{(1+b \Delta \lambda)^{2}}\left(r_{n}+\frac{\Delta \lambda}{\sqrt{1-\bar{D}_{n}}}\right)^{2}
\end{aligned}
$$

\subsection{Tangent Modulus Operator}

The linearization of Equation (17(a)) using the iterative Newton-Raphson method, requires the computation of the tangent stiffness matrix with a manner consistent with time discritization of the stress $\underline{\sigma}_{n+1}$ as discussed above.

From Equation (23) the stress at $t_{n+1}$ can be rewritten:

$$
\underline{\sigma}_{n+1}=2 \mu \sqrt{1-\bar{D}}\left[\sqrt{1-\bar{D}} \underline{e}_{n+1}^{*}-\Delta \lambda \underline{\tilde{n}}_{n+1}\right]
$$

The tangent matrix is then given by: 
$\frac{d \underline{\sigma}_{n+1}}{d \underline{\varepsilon}_{n+1}}=\frac{\partial \underline{\sigma}_{n+1}}{\partial \underline{e}_{n+1}^{*}}: \frac{\partial \underline{e}_{n+1}^{*}}{\partial \underline{\varepsilon}_{n+1}}+\frac{\partial \underline{\sigma}_{n+1}}{\partial \Delta \lambda} \otimes \frac{\partial \Delta \lambda}{\partial \underline{\varepsilon}_{n+1}}+\frac{\partial \underline{\sigma}_{n+1}}{\partial \underline{\tilde{n}}_{n+1}^{*}}: \frac{\partial \underline{\tilde{n}}_{n+1}^{*}}{\partial \underline{\varepsilon}_{n+1}}$

In which all the derivatives can be easy analytically calculated except the terms $\frac{\partial \Delta \lambda}{\partial \underline{\varepsilon}_{n+1}}$ and $\frac{\partial \underline{\tilde{n}}_{n+1}^{*}}{\partial \underline{\varepsilon}_{n+1}}$ which be obtained from the derivation of $\bar{f}_{n+1}$ Equation (30) performed the local Newton-Raphson procedure applied to $\bar{f}_{n+1}$.

The general organization chart of the program is shown to the Figure 1.

The theoretical model presented above, has been implemented in a finite elements program. The program was written using FORTRAN code. It has been written using the same format of the program developed by the international centre for numerical methods in engineering (CIMNE, Barcelona). This software is an adaptation of PLAST2 program developed by Owen and Hinton in their classical texts on finite element modelling [23]. Also, this program has been adapted for structural damage analysis. The finite element T3 is implemented with three degrees of freedom per node $(u, v, \bar{D})$ to solve the problem. The nodal variable $\bar{D}$ is systematically transferred to Gauss points to achieve the coupling with elastoplastic-damage. Once a Gauss point is completely damaged ( $\bar{D}=1)$, the corresponding element is removed from the calculation loop. The preparations of data as well the visualization of results are achieved with the help of the GID (graphical user interface for geometric modelling, data input, and visualization of results for all types of numerical simulation by CIMNE).

\section{Application}

The proposed damage-gradient based non local elastoplastic model is now used to predict the damage-plastic flow localization under simple plan strain tension. First an initially homogeneous plane strain tension test is performed, then a notched plane strain specimens is investigated with respect to the localization of plastic strain, damage and its dependence to the length scale parameter $(\omega)$.

\subsection{Initially Homogeneous Plane Strain Tension Test}

The tensile specimen is presented in Figure 2 with the initial homogenous mesh size $h=0.16 \mathrm{~mm}$. The boundary conditions which consist to apply a displacement along the "y" axis with $u=0$. The upper side of the specimen while the down side still completely clamped. The material constants are given in Table 1.

The first effect to be investigated is the effect of the internal length scale lying from $0.0 ; 0.3 ; 0.8,1.5$ and 3.0
Table 1. Board mechanical features.

\begin{tabular}{ccccccccc}
\hline $\begin{array}{c}\mathrm{E} \\
(\mathrm{MPa})\end{array}$ & $v$ & $\begin{array}{c}\sigma_{y} \\
(\mathrm{MPa})\end{array}$ & $\begin{array}{c}\mathrm{Q} \\
(\mathrm{MPa})\end{array}$ & $\mathrm{b}$ & $\beta$ & $\mathrm{S}$ & $\mathrm{s}$ & $\mathrm{Y}_{0}$ \\
\hline $21.10^{4}$ & 0.3 & 500 & 1000 & 40 & 1 & 10 & 0.8 & 0 \\
\hline
\end{tabular}

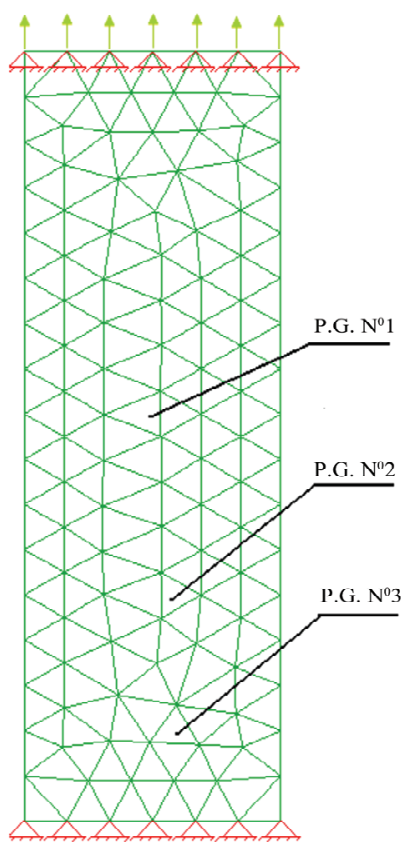

Figure 2. Mesh and boundary conditions applied to a part on a tensile test.

in the plastic damage flow localization using a fixed mesh with $\mathrm{h}=0.16 \mathrm{~mm}$.

In Figure 3 are summarized the global Force-displacement curves obtained with the five different values of $\omega$. Clearly and as expected, higher are the $\omega$ values, later is the fracture occurrence. The displacement at fracture varies from $u_{f r}=0.39 \mathrm{~mm}$ for $\omega=0.0$ (local model) to $u_{f r}=$ $0.71 \mathrm{~mm}$ for $\omega=3.0$. The spatial distribution of the

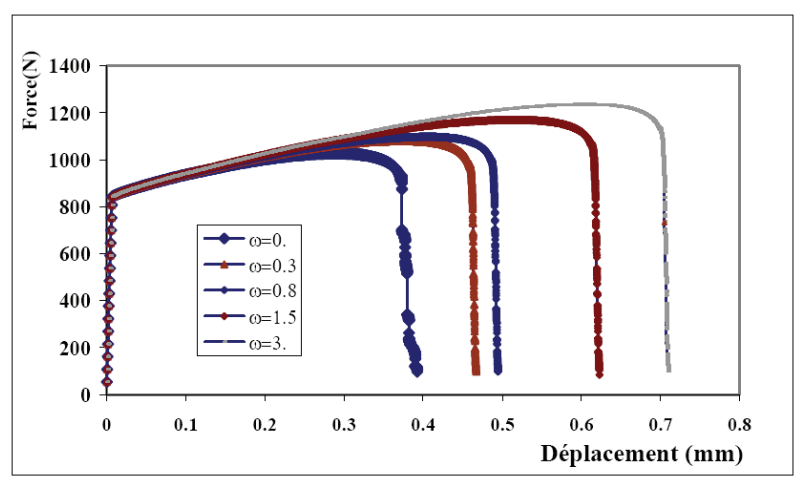

Figure 3. Force-displacement figure for different values of $\omega$. 
accumulated plastic strain and the ductile damage are shown in Figure 4 for $\omega=0.0, \omega=0.3$ and $\omega=1.5$ at three different values of the applied displacement $u=0.2 \mathrm{~mm}$, $u=0.3 \mathrm{~mm}$ and $u=0.39 \mathrm{~mm}$ corresponding to the fracture predicted by the local model with $\omega=0.0$. For $\omega=0.0$ (i.e. fully local model) the plastic strain and the damage localize more rapidly leading to the final fracture at $u_{f r}=$ $0.39 \mathrm{~mm}$ (see Figures 4(a, d, g)). Clearly the damage

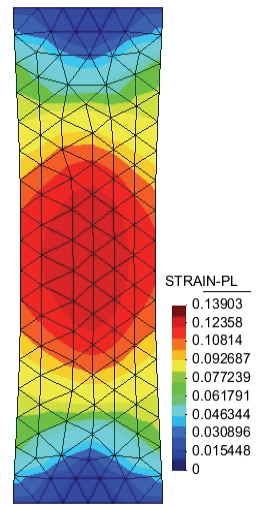

(a)

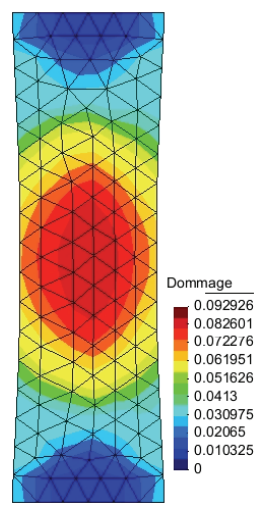

(a)
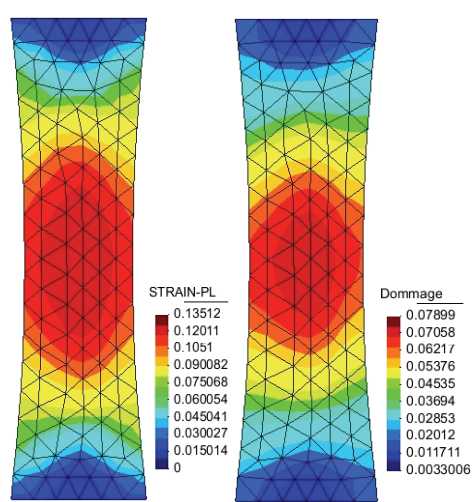

(b)

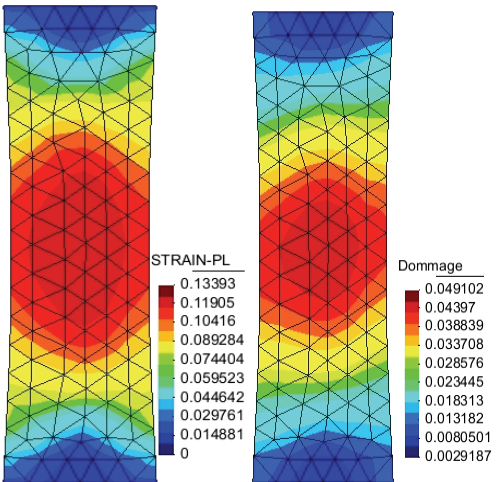

(c)

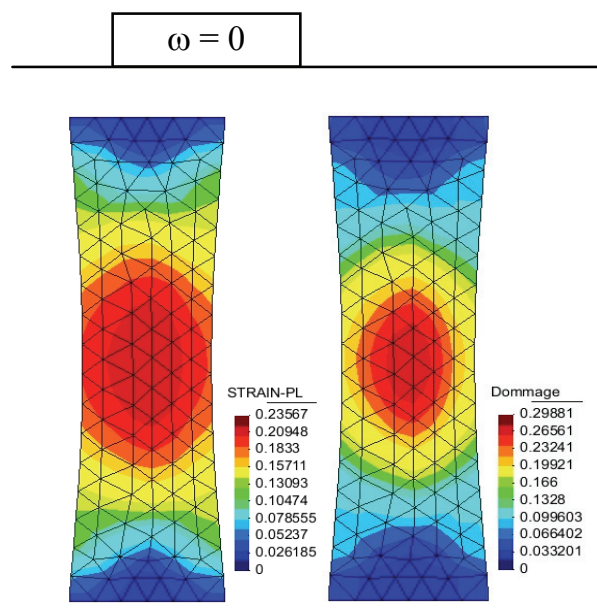

$\omega=0.33$ $u=0.2$
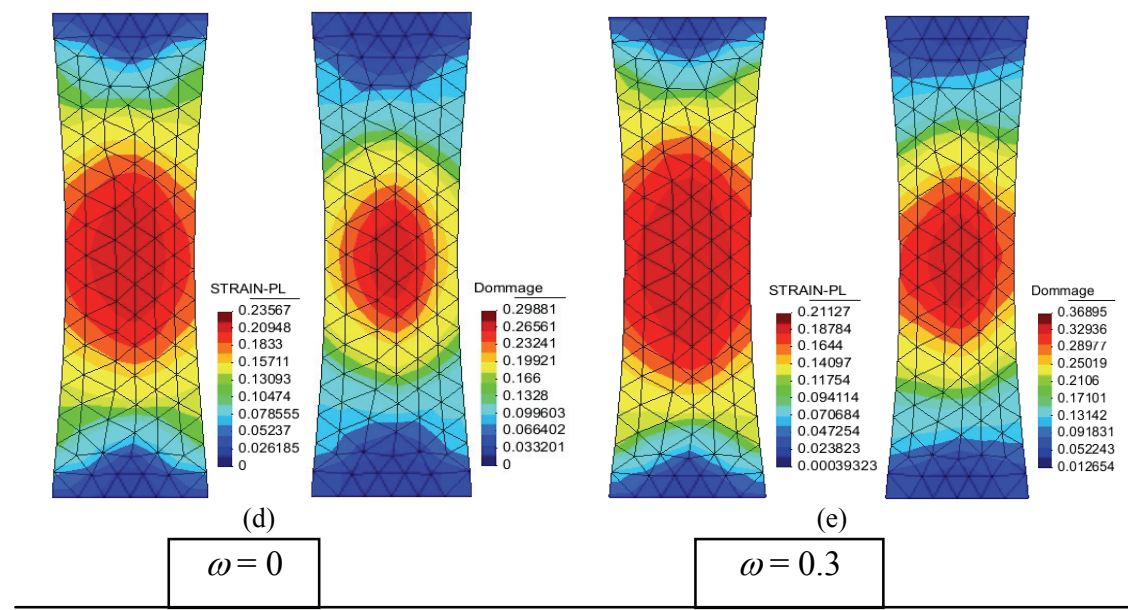

(e)

$\omega=1$

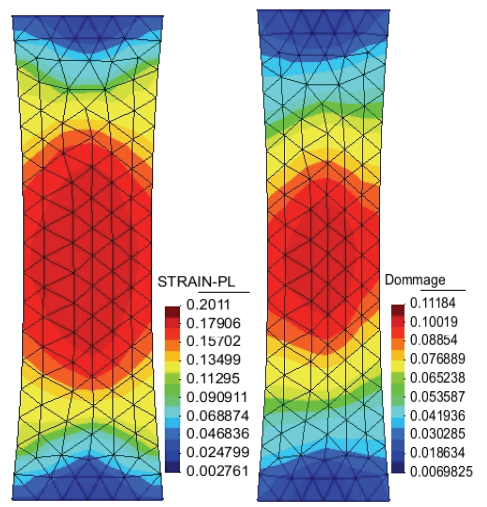

(f)

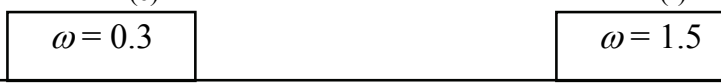

$u=0.3$

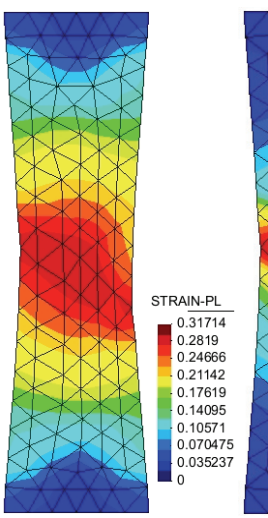

$\omega=0$

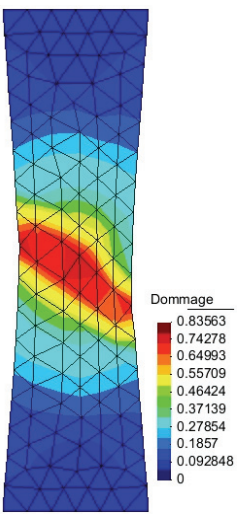

(g)
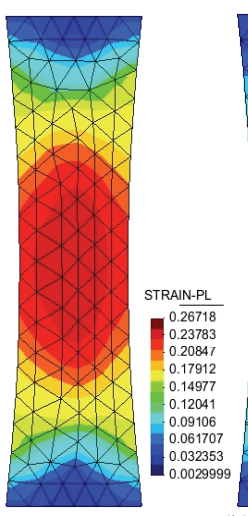

$\omega=0.3$

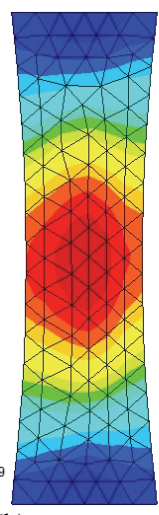

(h)

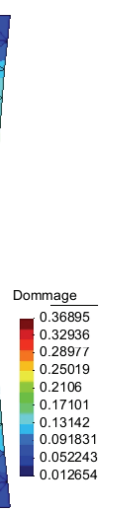

012654

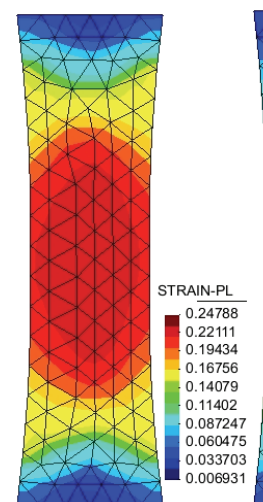

(j)

$\omega=1.5$ $\mathbf{u}=\mathbf{0 . 4}$

Figure 4. Distribution of plastic strain and damage at different displacement for different $\boldsymbol{e}$ for different values of $\omega$. 
zone localizes inside a single row of elements as indicated in Figure 4(g). For the some displacements the localization of the plastic strain and the damage taken place inside a more wide zones as can be seen in Figures $\mathbf{4}(\mathbf{b}, \mathbf{e}, \mathbf{h})$ for $\omega=0.3$ and Figures $\mathbf{4}(\mathbf{c}, \mathbf{f}, \mathbf{j})$ for $\omega=1.5$. Note that when $u=0.39$ corresponding to the finale fracture of the specimens for $\omega=0.0$ (local model), the maximum damage values are assured $\bar{D}_{\max }=0.36$ for $\omega=0.3$ and $\bar{D}_{\text {max }}=0.17$ for $\omega=1.5$ for from the final fracture condition $\bar{D}_{\max } \geq 0.999$. The final fracture obtained with the three values of " $\omega$ " can be taken from Figure 3 and the corresponding distribution of the mechanical fields are given in Figure 5. Clearly, the fully damaged zone (i.e. macroscopic crack) covers a large number of element when $\omega=0.3$ and $\omega=1.5$ (non local model) while for $\omega=0.0$ (local model) the crack width is limited to one element row.

These results indicate clearly the effect of the internal length scale in the elastoplastic solution with damage- induced softening. It is worth noting test one the $\omega$ is different from zero, the localization becomes mesh independent. Also, from Figure 5 the equivalent strain values approach zero inside the fully damaged zone. Clearly the crack paths seems more realistic for the fully local model (i.e. $\omega=0.0$ ) that with the non local model when $\omega>0$. In fact the macroscopic crack width follows the shear band for $\omega=0.0$, while it covers the wide zone located at the specimen center for $\omega>0.0$ (non local model). This is highly questionable from the fracture point of view. Finally, the local stress-plastic strain curves for three different gauss points defined in Figure 2, are given in Figure 6. For the local model (i.e. $\omega=0.0$ ) the evolution of the stress various the plastic strain is the some for the three points as long as the stress state is homogeneous inside the specimens. When the diffuse necking takes place first the point $\mathrm{N}^{\circ} 3$ becomes elastically unloads, while the point $\mathrm{N}^{\circ} 2$ and $\mathrm{N}^{\circ} 1$ continue to be plastically loaded (See Figure 6(a)). Finally, the point $\mathrm{N}^{\circ} 2$ transforms into elastic unloading while the point $\mathrm{N}^{\circ} 1$ contin-

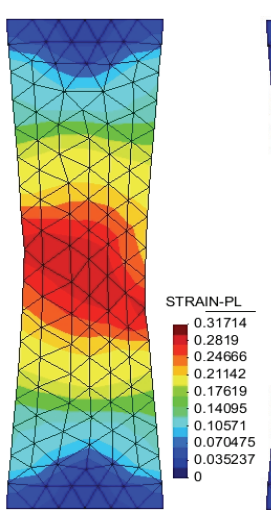

(a)

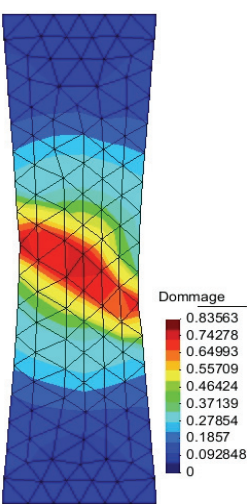

$\omega=0 \quad u_{f r}=0.39 \mathrm{~mm}$

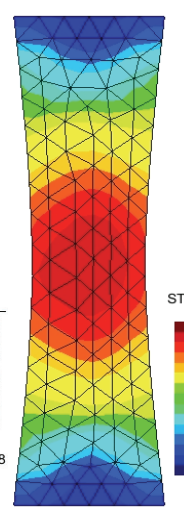

(b)
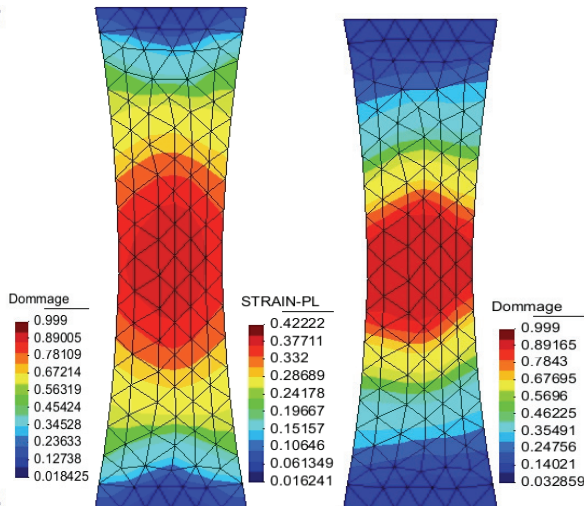

(c)

$\omega=0.3 \quad u_{f r}=0.47 \mathrm{~mm}$

$\omega=1.5 \quad u_{f r}=0.63 \mathrm{~mm}$

Figure 5. Respective distribution of, the plastic strain and at failure for different values of $\omega$.

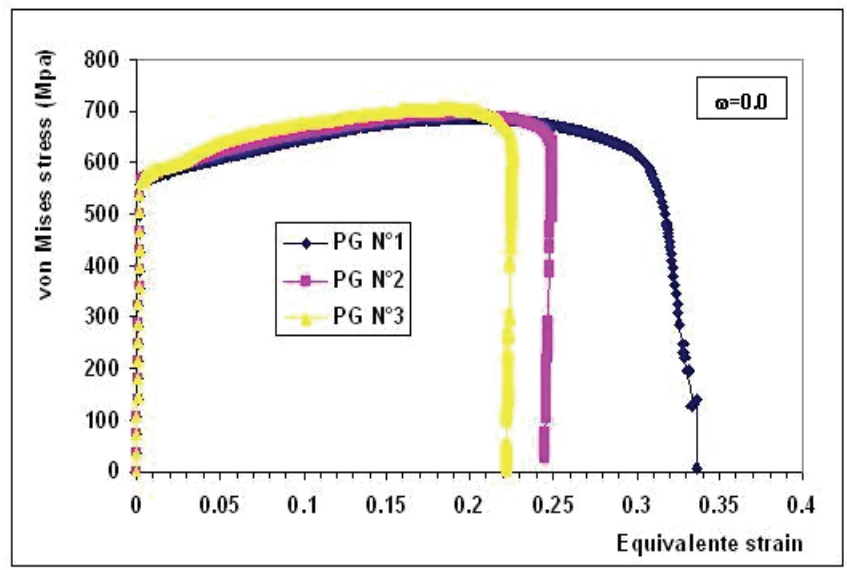

(a) 


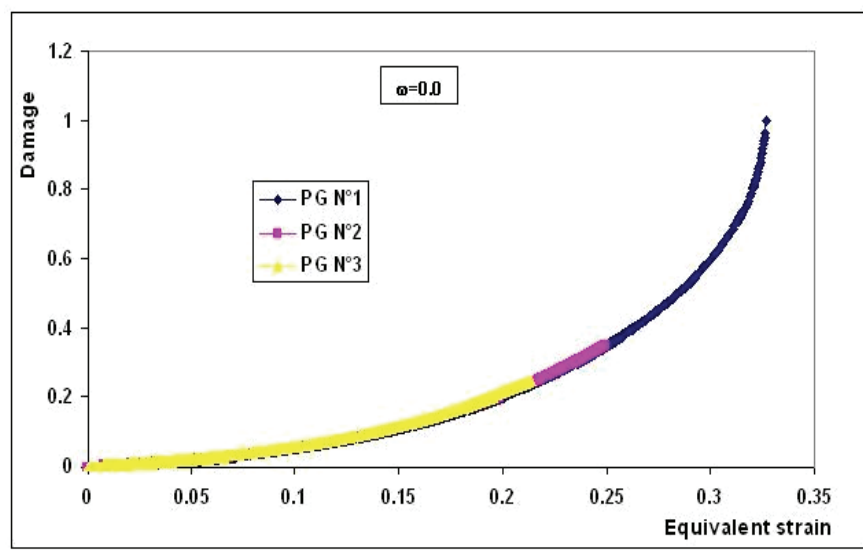

(b)

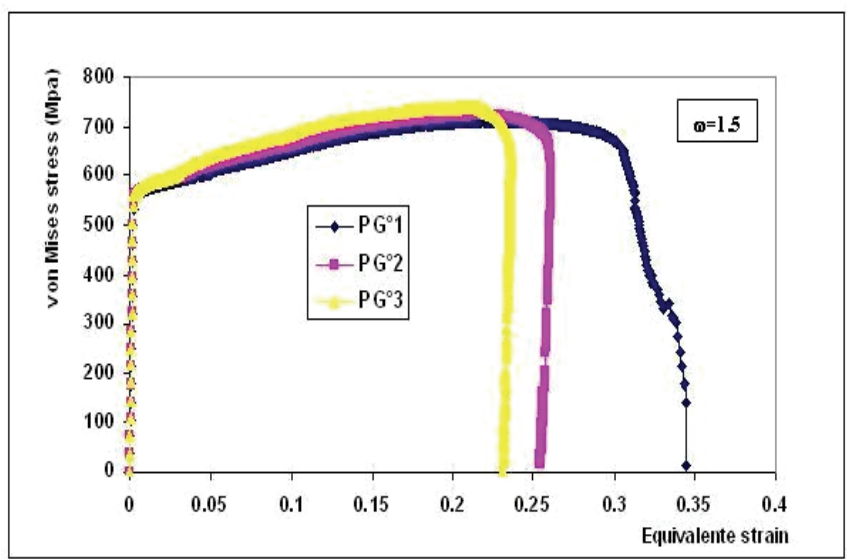

(c)

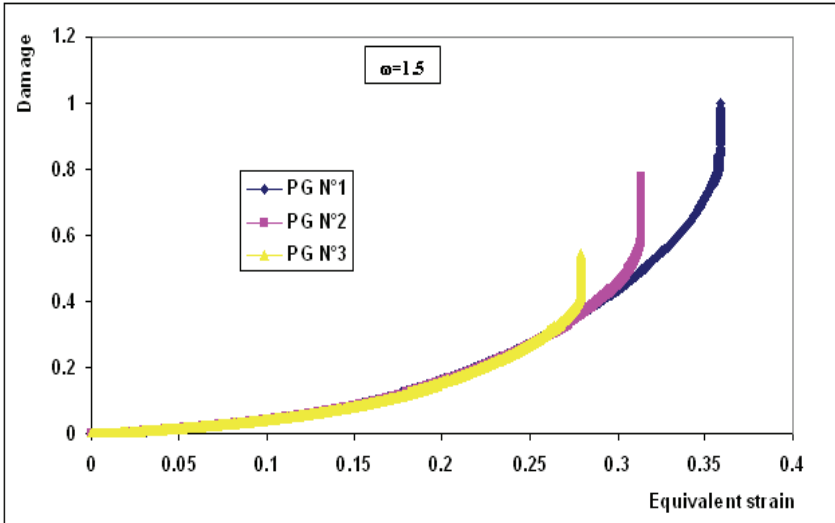

(d)

Figure 6. The evolution of the von Mises stress and the damage depending on the equivalent plastic strain for $\omega=0.0$ and $\omega=$ 1.5.

ues to be plastically loaded until elastic unloading while the point $\mathrm{N}^{\circ} 1$ continues to be plastically loaded until the final fracture. The corresponding damage evolves until $D$ $=1$ for point $\mathrm{N}^{\circ} 1$ while it saturates at $D=0.35$ for point $\mathrm{N}^{\circ} 2$ and $D=0.24$ for point $\mathrm{N}^{\circ} 3$, where are never damage evolution is observed since they are elastically unloaded. For the non local (i.e. $\omega=1.5$ ) the stress-plastic strain curves for three points behave similar to the local model according to the needing effect (Figure 6(c)). However, the damage evolves differently without reaching the final 
fracture for point $\mathrm{N}^{\circ} 3\left(D_{\max }=0.77\right)$ and the point $\mathrm{N}^{\circ} 2$ $D_{\max }=0.54$ ) while the finale fracture occurs for the point $\mathrm{N}^{\circ} 1$ as can be seen in Figure 6(d). Note that a constant value of $\omega$ with different values of the mesh size, the stress-plastic strain curves have been shown independent from the mesh size as can be fixed in [24]. This aspect is user investigated using double notched specimen.

\subsection{Notched Specimen}

Consider the saves notched specimen as investigated by Peerlings [3] and Nedjar [25] and shown in Figure 7. Three meshes are considered account the notched zone with $h=0.8, h=0.4 \mathrm{~mm}$ and $h=0.2 \mathrm{~mm}$. The some material constants of table $\&$ are used and two values of $\omega$ are investigated: $\omega=0.0$ (local model) and $\omega=1.0$.

In Figure 8 are shown the distribution of the plastic, the damage and the stress at the finale fracture for the three mesh sizes with $\omega=0.0$ (local model). Clearly the fully damaged zone is limited to one row of elements for this fully local. However, for the non local model $(\omega=$

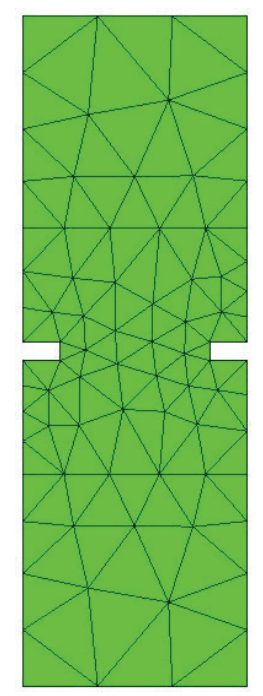

$h=0.8$

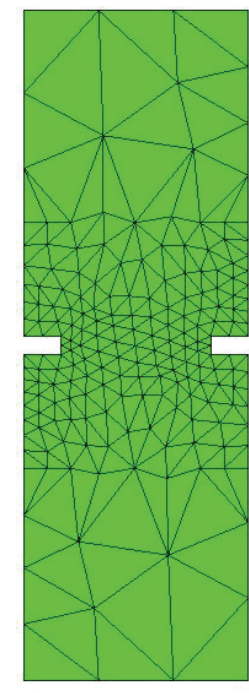

$h=0.4$

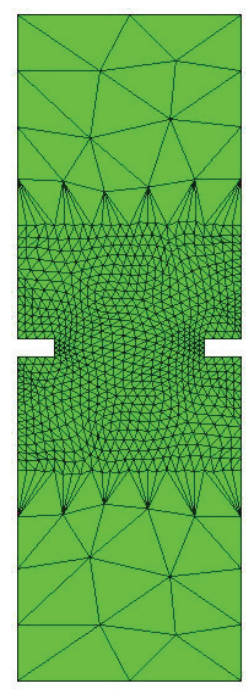

$h=0.2$
Figure 7. The notched plate, the Boundary conditions, the load type and size of the mesh studies at Neighbourhood notch.

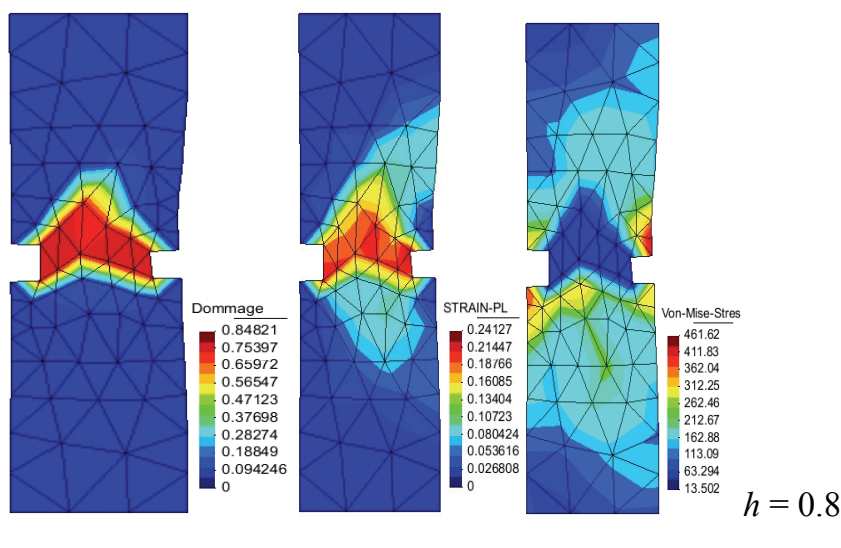

(a) $\omega=0 \quad u_{f r}=0.8 \mathrm{~mm}$

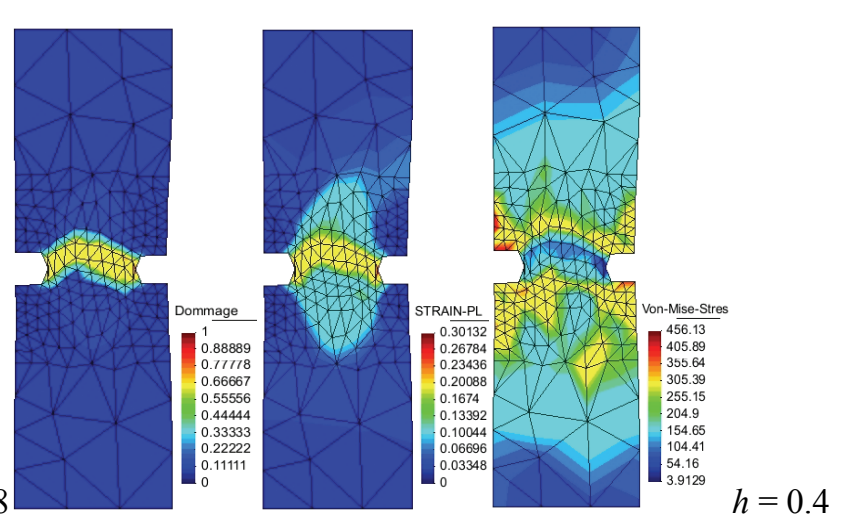

(b) $\omega=0 u_{f r}=0.9 \mathrm{~mm}$
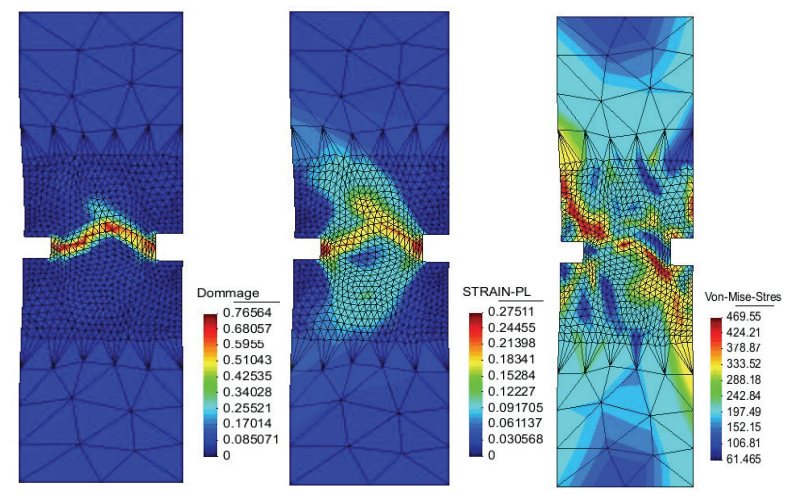

(c) $\omega=0 \quad u_{f r}=1.05 \mathrm{~mm}$

Figure 8. Respective distribution of the damage, the plastic deformation and the stress of von Mises at failure for $\omega=0$. 


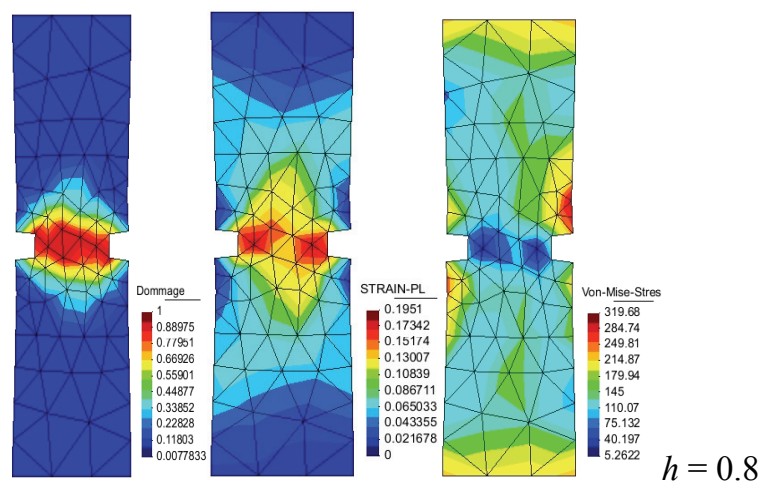

(a) $\omega=1 u_{f r}=1.275 \mathrm{~mm}$

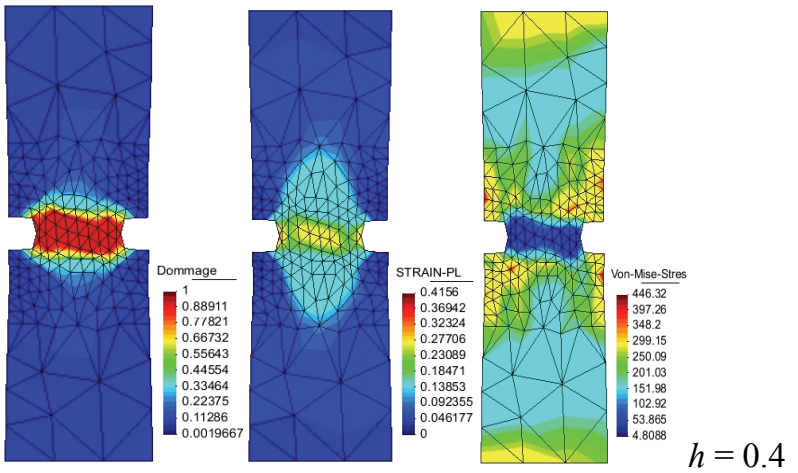

(b) $\omega=1 u_{f r}=1.27$

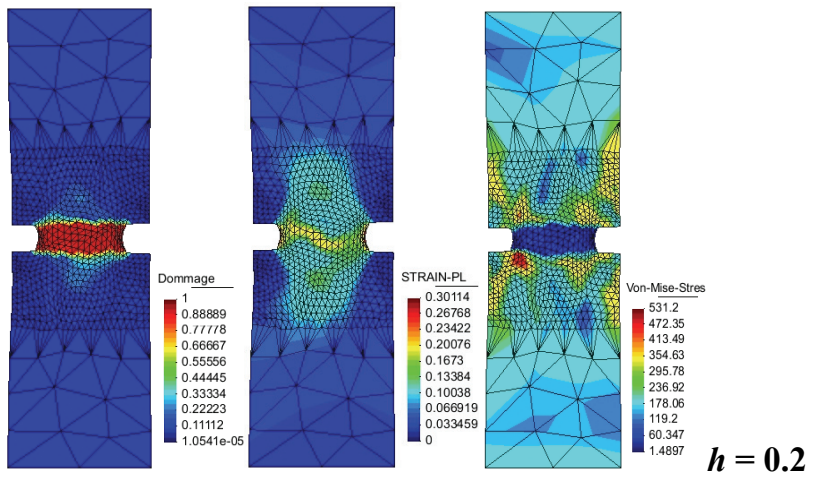

(c) $\omega=1 u_{f r}=1.27 \mathrm{~mm}$

Figure 9. Respective distribution of the damage, the plastic deformation and the stress of von Mises at failure for $\omega=1$.
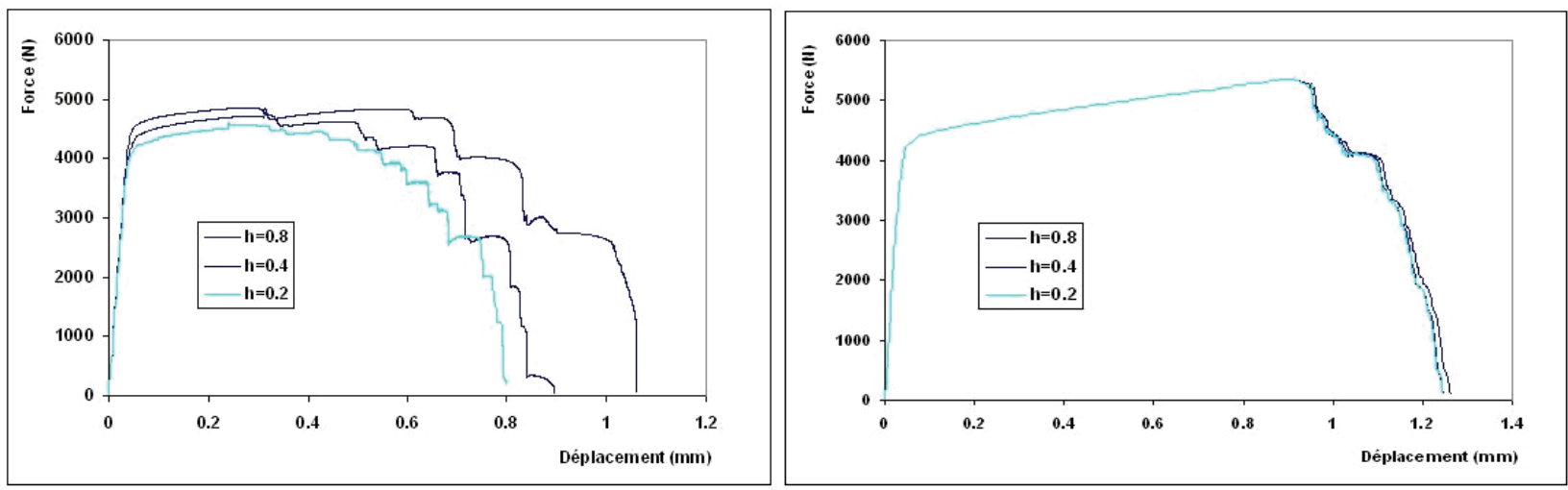

Figure 10. Global response for (a) case $\omega=0$ and (b) case $\omega=1$.

$1.0)$, we observe, that the fully damaged zone is limited to use row of element for the coarse mesh $h=0.8$ (Figure 9(a)) while it develops over many rows of elements for the first mesh as indicated in Figures 9(b) and 9(c). The global force-displacement curves obtained with the three meshes indicated clearly strong dependency to the mesh size for the local model (Figure 10(a)) and are independent from the mesh size for the non local model
(Figure 10(b)).

\section{Conclusions}

A simple damage-gradient elastoplastic model with non linear isotropic hardening has been developed and implemented into an in-horse finite element program. When applied to a simple plane strain tensile test of initially 
homogeneous and initially notched specimen, the model show a clear independence of the results on the mesh size when the internal length scale is non zero. This model will be implanted into a general propose Finite elements program in order to show its ability to give a mesh independent solution for more complex structures as in metal forming. Also the extension to the 3D structures is under program.

\section{References}

[1] G. Pijaudier-Cabot and Z. Bažant, "Nonlocal Continuum Damage, Localization Instability and Convergence," International Journal of Applied Mechanics, Vol. 55, No. 4, 1988, pp. 287-293.

[2] K. Saanouni, "Sur l'Analyse de la Fissuration des Milieux Elasto-Viscoplastique par la Théorie de l'Endommagement Continue," Thèse de Doctorat, Université de Technologie de Compiègne, 1988.

[3] T. Svedberg and K. Runesson, "An Adaptive Finite Element Algorithm for Gradient Theory of Plasticity with Coupling to Damage," International Journal of Solids Structures, Vol. 37, No. 48-50, 2000, pp. 7481-7499.

[4] R. H. J. Peerlings, "Enhanced Damage Modelling for Fracture and Fatigue," Ph.D. Dissertation, Technische Universiteit Eindhoven, 1999.

[5] C. Comi, "A Nonlocal Model with Tension and Compression Damage Mechanics," European Journal of Mechanics A/Solids, Vol. 20, No. 1, 2001, pp. 1-22.

[6] M. Geers, R. Ubachs and R. Engelen, "Strongly NonLocal Gradient-Enhanced Finit Strain Elastoplasticity," International Journal for Numerical Methods in Engineering, Vol. 56, No. 14, 2003, pp. 2039-2068.

[7] R. Abu-Al-Rub and G. Voyiadjis, "A Physically Based Gradient Plasticity Theory," International Journal of Plasticity, Vol. 22, No. 4, 2006, pp. 654-684.

[8] K. Saanouni and J.-L. Chaboche, "Comprehencive Structural Integrity," Vol. 10, 2003.

[9] R. Mindlin and N. Eshel, "On First Strain Gradient Theories in Linear Elasticity," International Journal of Solids and Structures, Vol. 4, No. 1, 1968, pp. 109-124.

[10] P. Germain, "The Method of Virtual Power in Continuum Mechanics, Part 2: Microstructure," SIAM Journal on Applied Mathematics, Vol. 25, No. 3, 1973, pp. 556-575.

[11] G. Maugin, "Nonlocal Theories or Gradient-Type Theories: A Matter of Convenience," Archives of Mechanics, Vol. 31, No. 1, 1979, pp. 15-26.

[12] S. Forest and R. Sievert, "Elastoviscoplastic Constitutive Frameworks for Generalized Continua," Acta Mechanica,
Vol. 160, No. 1, 2003, pp. 71-111.

[13] A. Eringen, "Microcontinuum Field Theories: Vol. I Foundations and Solids; Vol. II Fluent Media," Springer, New York, 1999.

[14] R. Chambon, D. Caillerie and T. Matsuchima, "Plastic Continuum with Microstructure, Local Second Gradient Theories for Geomaterials," International Journal of Solids and Structures, Vol. 38, 2001, pp. 8503-8527.

[15] S. Forest, "Micromorphic Approach for Gradient Elasticity, Viscoplasticity and Damage," Journal of Engineering Mechanics, Vol. 135, No. 3, 2009, pp. 117-131.

[16] R. Peerlings, T. Massart and M. Geers, "A Thermodynamical Motivated Implicit Gradient Damage Framework and its Application to Brick Masonry Cracking," Computer Methods in Applied Mechanics and Engineering, Vol. 193, No. 30-32, 2004, pp. 3403-3417.

[17] K. Saanouni, C. Forster and F. Ben-Hatira, "On the Anelastic Flow with Damage," International Journal of Damage Mechanics, Vol. 3, No. 2, 1994, pp. 140-169.

[18] K. Saanouni, H. Badreddine and M. Ajmal, "Advances in Virtual Metal Forming Including the Ductile Damage Occurrence: Application to 3D Sheet Metal Deep Drawing," Journal of Engineering Materials and Technology, Vol. 130, No. 2, 2008, 1-11.

[19] D. Sornin, "Sur les Formulations Elastoplastiques Non Locales en Gradient d'Endommagement," Thèse de Doctorat, Université de Technologie de Troyes, 2007.

[20] S. Boers, P. Schreurs and M.Geers, "Operator-Split Damage-Plasticity Applied to Groove Forming in Food Can Lids," International Journal of Solids and Structures, Vol. 42, No. 14, 2005, pp. 4154-4178.

[21] A. Simone, G. Wells and L. Sluys, "From Continuous to Discontinuous Failure in a Gradient-Enhanced Continuum Damage Model," Computer Methods in Applied Mechanics and Engineering, Vol. 192, No. 41, 2003, pp. 4581-4607.

[22] Y. Hammi, "Simulation Numérique de l'Endommagement dans les Procédés de Mise en Forme," Université de Technologie de Compiègne, 2000.

[23] D. R. J. Owen and E. Hinton, "Finite Elements in Plasticity, Theory and Practice," Pineridge Press Limited, Swansea, 1988.

[24] M. Almansba, K. Saanouni and N. E. Hannachi, "Régularisation d'un Modèle Elastoplastique par Introduction d'un Gradient d'endommagement," XIX ${ }^{\text {ème }}$ Congrès Français de Mécanique, CFM'09, France, 2009.

[25] B. Nedjar, "Elastoplastic-Damage Modelling Including the Gradient of Damage: Formulation and Computational Aspects," International Journal of Solids and Structures, Vol. 38, No. 30-31, 2001, pp. 5421-5451. 